\title{
Special Collection on New Methods and Applications of Optical and Photo-Mechanics
}

\author{
W. Qiu ${ }^{1} \cdot$ H. M. Xie ${ }^{2} \cdot$ X. L. Gong ${ }^{3}$ \\ () Society for Experimental Mechanics 2021
}

Experimental mechanics is one of the important pillars for the development of modern mechanics and related disciplines. Optical/photo-mechanics is a key branch of experimental mechanics, and has been widely used to characterize the mechanical behavior of materials and structures. These methods of measurement and their applications have drawn great attentions from researchers for many years.

It is our pleasure to present the fourteen papers in this special issue, entitled "New methods and applications of optical/photo-mechanics". The special issue attempts to focus on the latest developments in the field of optical/photomechanics in China, which covers a range of topics to reflect recent advances in optical methods including digital image correlation (DIC), projection moiré, electron moiré, electron backscattered diffraction (EBSD) and synchrotron radiation computed tomography (SR-CT), etc. A brief introduction of these papers is shown as follows:

For realizing accurate strain measurement in spaceconstrained scenarios, B. Dong et al. improved the 2D-DIC to provide quality displacement and strain measurement by combining built-in coaxial illumination and telecentric fluorescent imaging. Real uniaxial tensile test and thermal strain measurement test in space-constrained and challenging scenarios verified the accuracy and practicality of the proposed fluorescent 2D-DIC. With the improved performance, compactness, and ease of use, this fluorescent 2D-DIC is expected to be widely applied in future research.

\section{L. Gong}

gongxl@ustc.edu.cn

1 Tianjin Key Laboratory of Modern Engineering Mechanics, School of Mechanical Engineering, Tianjin University, Tianjin 300350, China

2 School of Aerospace Engineering, Tsinghua University, Beijing 100081, China

3 CAS Key Laboratory of Mechanical Behavior and Design of Materials, Department of Modern Mechanics, University of Science and Technology of China, Hefei 230026, Anhui, China
In order to solve the problem of DIC measurement in an electrochemical environment, H.M. Xie et al. developed a fluorescence DIC strain measurement method characterized by fluorescent speckle and active optical imaging. Combined with electrochromic-based Li concentration detection, the spatiotemporal evolution of strain and $\mathrm{Li}$ concentration of a graphite electrode were measured. This work overcomes the difficulties of fine strain measurement and collaborative concentration characterization during the electrochemical process, providing an effective experimental method for further exploration of mechano-electro-chemical coupling.

To realize closed loop control of systems under large deformation and rotation, a real-time 3D-DIC method was proposed by X.X. Shao and X.Y. He. A temporal-spatial deformation transfer scheme and the improved interpolation look-up table were established for efficient temporal and stereo matching. Benefiting from the efficient IC-GN algorithm and parallel processing software they developed, real-time multipoint deformation measurement at $60 \mathrm{~Hz}$ with 50 points and full-field deformation measurement at $15 \mathrm{~Hz}$ with 5000 points were realized. In their future work, they plan to provide a real-time and fully automatic deformation measurement sensor for applications in scientific and engineering fields.

Camera calibration is an essential step in the computer vision-based 3D shape and deformation field measurements. In terms of direct linear transformation of the lines (DLT-Lines), Z.C. Shi et al. present a full camera calibration method to estimate the camera parameters, including camera intrinsic parameters, extrinsic parameters and lens distortion parameters, from a single image with six or more noncoplanar lines. Both synthetic and real data validated that the proposed method could provide competitive calibration results, which demonstrates its applicability in the work site.

Ratcheting is an important mechanical behavior of metals and alloys. H. Jiang et al. proposed a nonlinear virtual field method to characterize the ratcheting effect and identify the corresponding kinematic constitutive rules. The parameters 
of the Chaboche constitutive equation were inverted from strain field data. Numerical experiments show that the virtual field method can effectively identify ratchet evolution characteristics in inhomogeneous deformation fields. By training the measured field data from 3D-DIC, it is shown that the algorithm can also run effectively under the complex working conditions of non-uniform deformation.

In order to meet the requirement of projection moiré method in engineering applications, based on an iterative calculation model and the self-calibration method, T. Cai et al. established an arbitrary arranged projection moiré system for accurate and convenient 3D surface measurement. The parameters of the projection system and reception system were optimized simultaneously. Both simulations and experiments were used to validate the feasibility and accuracy. Results show that the proposed methods can reduce the systematic error and simplify the complexity of operations effectively.

DIC's measurement accuracy is mainly determined by the image acquisition system and the quality of the speckle pattern. The objective of the work by Y. Wang et al. was to improve the measurement accuracy through optimization of aperture and speckle pattern. They found the optimal aperture and speckle generation parameters (speckle diameter and density) through evaluating the measurement errors with different apertures and different speckle generation parameters. These results could offer an important reference for the selection of parameters in actual engineering applications.

To improve DIC's measurement accuracy, knowing the cause of various errors is necessary. Based on Fourier analysis, Z. Wang et al. established a frequency-domain error model for 2D-DIC. This model can analyze the noiseinduced random error, the interpolation-induced systematic error, as well as their combination. Its validity was verified by both theoretical analysis and numerical experiments. This model reveals the relation between the systematic error and random error, which could be used to evaluate the quality of images for DIC analysis.

In the synchrotron radiation computed tomography (SRCT) analysis under mechanical loading process, accurate reconstruction of finite angle projection information shielded by mechanical loading devices is a bottleneck problem. J.W. $\mathrm{Li}$ et al. proposed a novel experimental strategy to improve the results of in situ SR-CT mechanical loading test, called TSIR-CT. From the new perspective of the frequency domain, the tilt sampled projection information was utilized to recover the missing angle information. The proposed method was validated by a series of numerical reconstruction experiments and actual SR-CT data. The results lay a foundation for the quantitative analysis of the deformation field using SR-CT data.

Metal additive manufacturing has huge application prospects in the field of aerospace, precision instruments, and biomedical engineering etc..... However, the low manufacturing quality of key components is a bottleneck restricting its further development and application. Online stress detection in manufacturing process is very important for quality control. G.Z. Zeng developed a hybrid characterization method for the online evaluation of stress fields during laser cladding. The method combined realtime temperature field measurements, three-dimensional deformation field measurements and finite element calculations, which realized the online characterization of three-dimensional stress field in the laser engineered net shaping (LENS) printing process, providing important experimental techniques and data for the quality control of $3 \mathrm{D}$ printing.

In addition to the above-mentioned results on the method, technique and system on optical/photo-mechanics, this special issue also includes some applications in frontier and interdisciplinary research. To study the mechanism of highvelocity oxy-fuel (HVOF)-sprayed metallic coatings, F.C. Lang et al. investigated the deformation field of stainless steel induced by the impact of an individual particle. The microscopic deformation field on the surface induced by the impact of micro-particles with a diameter of $18 \mu \mathrm{m}$ was determined using the electron moire method and numerical simulation. The results show that the electron moire technique can be a useful tool for the measurement of the deformation field induced by an impacted particle in a very local area with a size on the order of microns.

Current fundamental research requires more precise measurement of internal microstructure and deformation in materials. C. Zhao and X. Li characterized the deformation of two iron-based alloys undergoing bending testing through high resolution electron backscattered diffraction (HR-EBSD) and high-resolution digital image correlation (HR-DIC). The residual elastic strain, geometrically necessary dislocation density (GND) and plastic strain distributions were obtained. The results show that grain/twin boundaries and carbides resulted in GND density increase, promoted work hardening and accumulated high residual elastic strain. Heterogeneous grain/carbide size distribution leaded to stress concentration and caused carbide decohesion and brittle fracture.

For the large flexible cable-net structure, traditional videometric methods cannot be used in the measurement for its large overall size and small partial size. S. Qin et al. proposed a novel videometric method to measure the topography and deformation of a large cable net structure by using tiny-light spot markers. The relationship between the imaging parameters and the gray distribution of the light spot markers were derived and verified. The 3D tomography measurements of a large curved surface and a flexible cable net demonstrated the effectiveness of the proposed videometric method. 
Vortex stability is critically important for applications of type-II superconductors; however, the existing research has not addressed the problem of the power threshold value (PVT) for activating a flux avalanche. C. Wang et al. constructed two systems to investigate the influences of temperatures, applied magnetic fields and laser activating sites on the PTV of $\mathrm{YBa}_{2} \mathrm{Cu}_{3} \mathrm{O}_{7-\mathrm{x}}(\mathrm{YBCO})$ thin films, and to find the relationship between avalanche velocities and laser activating sites. Two models were provided to depict the dependence of PTV on temperature and avalanche velocity on activating sites, respectively. This result is of significance to evaluate the flux stability for practical applications of high temperature superconducting films.

In sum, the papers in this special issue aim to report the latest progress of methods and techniques on optical/photomechanics and relative applications. The topics include morphology and deformation measurement with 2D-DIC, 3D-DIC, fluorescence DIC, HR-DIC, SR-CT, HR-EBSD, projection moiré, electron moiré et al. The key issues such as imaging quality, calibration methods and error analysis are analyzed. And these optical/photo-mechanical methods are applied to actual engineering and interdisciplinary field, which should be of interest to researchers in mechanics, materials and other related fields. With the developments and requirements of engineering and interdisciplinary problems, we hope that this special collection can provide a framework and a direction to further promote the application of optical/photo-mechanics.

Finally, we would like to express our sincere gratitude to all of the contributing authors, the editorial team of Experimental Mechanics, and the Society for Experimental Mechanics. Without their contributions and support, this special collection could not have been successfully published. We also appreciate the reviewers for their invaluable assistance during the review process of all papers in this special collection.

Publisher's Note Springer Nature remains neutral with regard to jurisdictional claims in published maps and institutional affiliations. 